\title{
Neuropsicología de la memoria declarativa asociada a contenido emocional en enfermedad de Parkinson ${ }^{*}$
}

\author{
Neuropsychology of declarative memory associated with the emo- \\ tional content in Parkinson's disease
}

\section{Neuropsicología da memoria declarativa associada ao conteúdo emocional na doença de Parkinson}

\author{
Jorge Alexander Ríos-Flórez \\ Estiven Del Valle Del Valle ${ }^{* * *}$ \\ Claudia Marcela Marín Ramírez \\ Sara Agudelo Montoya $a^{* * * * *}$ \\ Andrea Alexandra Toro Londoño
}

Recibido 29. 02. 2016 • Arbitrado 15. 03. 2016 • Aprobado 20.04. 2016

\section{Resumen}

El objetivo de la investigación fue caracterizar el funcionamiento de la memoria declarativa, asociada al contenido emocional, en adultos mayores con enfermedad de Parkinson, para lo cual, se aplicó una

* $\quad$ Artículo producto del macroproyecto "Evaluación de la memoria declarativa asociada a contenido emocional en el envejecimiento normal y patológico", formulado en la línea de investigación neuroenvejecimiento y demencias, vinculada al grupo de estudio e investigación en neurociencias Hippocampus. Fecha de inicio febrero de 2015; fecha de finalización noviembre de 2015.

** Investigador, especialista en evaluación y diagnóstico neuropsicológico, magíster en neuropsicolología clínica. Director del grupo de estudio e investigación en neurociencias Hippocampus. Docente, Universidad Politécnico Grancolombiano, Tecnológico de Antioquia Institución Universitaria, Universidad Minuto de Dios, Medellín, Colombia. alexanderriosflorez@gmail.com / hippocampus geei@gmail.com.

*** Psicólogo - Investigador, miembro del grupo de estudio e investigación en neurociencias Hippo campus, Medellín, Colombia. estivendd@hotmail.com.

**** Psicóloga - Investigadora, miembro del grupo de estudio e investigación en neurociencias Hippo campus, Medellín, Colombia. clamarg1@hotmail.es.

***** Psicóloga - Investigadora, miembro del grupo de estudio e investigación en neurociencias Hi ppocampus, Medellín, Colombia. sara.agudelomo@gmail.com.

*******Psicóloga - Investigadora, miembro del grupo de estudio e investigación en neurociencias Hippocampus, Medellín, Colombia. andreatl89@gmail.com. 
batería de pruebas, en las que se evaluó atención, concentración, los almacenes de memoria y diversos tipos de emociones; se tomó una muestra de 40 participantes, 20 dentro de envejecimiento normal, y 20 con enfermedad de Parkinson; la mitad de cada grupo presenció la versión neutra de una prueba de memoria, asociada a contenidos emocionales; y la otra mitad, la versión emocional. Los pacientes con enfermedad de Parkinson reportaron mayor carga emocional, relacionada con sus recuerdos, cuando se han codificado y almacenado, ante situaciones altamente emocionales. Así mismo, emociones como felicidad y sorpresa no se asocian a la información evocada por estos pacientes. De otro lado, la información carente de contenido emocional, se codifica y almacena de igual manera ante la condición de envejecimiento normal o patológico.

Palabras clave: Adulto mayor, deterioro cognitivo, enfermedad de Párkinson, emociones, envejecimiento, memoria declarativa.

\section{Abstract}

The aim of this research was to characterize the operation of declarative memory associated with emotional content in old people suffering from Parkinson's disease. In order to do this, a test battery in which the attention, concentration, memory stores, and several types of emotions were assessed, was carried out. A sample of 40 participants was taken, 20 among the normal aging and 20 suffering from Parkinson's disease. Half of each group took the neutral version of the memory test associated with emotional contents, the other half did it with the emotional version. Patients with Parkinson's disease showed higher emotional content related to their memories when being encoded and stored under highly emotional situations. In addition, emotions such as joy and surprise are not associated with information evoked by these patients. On the other hand, the information with a lack of emotional content is encoded and stored similarly under condition of normal or pathological aging. 
Keywords: Elderly, cognitive impairment, Parkinson's disease, emotions, aging, declarative memory.

\section{Resume}

O objetivo da investigação foi caracterizar o funcionamento da memoria declarativa, associada ao conteúdo emocional em adultos maiores com a doença de Parkinson, pelo qual, se aplicou uma bateria de provas, nas quais se avaliou a concentração, armazenamento da memoria e diversos tipos de emoções; a mostra foi realizada em 40 participantes, 20 dentro do envelhecimento normal e 20 com a doença de Parkinson; a metade de cada grupo presenciou a versão neutra de um teste de memoria, associada aos conteúdos emocionais; e a outra metade a versão emocional. Os pacientes com a doença de Parkinson mostraram maior carga emocional, relacionada com as suas lembranças, quando são codificadas e armazenadas em situações altamente emocionais. Assim mesmo, as emoções como felicidade e surpresa não se associam na informação transmitida por estes pacientes. Por outro lado, a informação carente de conteúdo emocional, se codifica e armazena de igual maneira que a condição do envelhecimento normal ou patológico.

Palavras chave: Adulto maior (idoso), deterioro cognitivo, doenca de Parkinson, emoções, envelhecimento, memoria declarativa.

El cerebro, como el universo, tiene tantos rincones inexplorados que con cada nuevo avance nos acerca a la comprensión de un asunto y nos aleja de la certeza absoluta.

[Alexander Ríos] 


\section{Introducción}

La literatura científica, como lo refiere Ríos (2016), reporta ampliamente el desarrollo de investigaciones que interrelacionan el funcionamiento de la memoria con las emociones, y la influencia de éstas en la codificación de la información; sin embargo, se han centrado su foco en la creación de "papel científico" sin propósitos aplicados de los resultados, cuyos datos deberían tener por objeto el campo clínico en la rehabilitación de las poblaciones objeto de las investigaciones; este propósito motiva y orienta a generar propuestas nuevas de estudios que posibiliten la intervención del sujeto.

En este sentido, a lo largo de décadas de investigación se ha abordado la demencia como patología del envejecimiento, y se le ha prestado mayor atención, teniendo en cuenta que la población mundial tiende a envejecer. Particularmente, los datos son generales y no se centran en una patología en particular, como lo es para el caso del deterioro cognitivo leve, la demencia multi-infarto y la tercera causa de demencia más frecuente, asociada a la enfermedad de Parkinson (Vargas, 1997). En torno a ésta última, diversos autores (Micheli, 2006; Casamitjana, 2007; García, Sauri Suárez, Meza Davalo y Lucino Castillo, 2008), refieren que los síntomas más frecuentes en los pacientes, son temblores en reposo, bradiscinesia, rigidez y alteración de los reflejos posturales, y se describió, por primera vez, por James Parkinson, en Inglaterra en 1817, bajo el nombre de parálisis agitante.

Sin embargo, otros estudios (Muñiz Casado y Rodríguez Fernández, 2007; Parrao-Díaz, Chaná-Cuevas, Juri-Claverías, Kunstmann y Tapia-Núñez, 2005; Meza, 1998; Vila y Barbero, 1992), plantean qué en la esfera cognitiva, la Enfermedad de Parkinson no solo presenta síntomas de tipo motor, sino que cubre un gran espectro que, desde fases iniciales, se manifiesta en déficit en las esferas autonómica y cognitiva, hasta llegar a demencia; la afectación cognitiva se centra en las funciones ejecutivas y las memorias, principalmente, en la cual se ve afectado el recuerdo libre, a corto y largo plazo, por canales verbal y visual, y el compromiso en la manipulación de conocimiento adquirido y su evocación. 


\section{Referentes Teóricos}

\section{Las memorias y su afectación en deterioro patológico}

El mayor compromiso de las patologías, a nivel de envejecimiento, es de la memoria, entendida como un sistema de adquisición, almacenamiento y recuperación de la información, obtenida a través de los sentidos; desde la neuropsicología, se define como una función superior netamente cognitiva, la cual permite registrar, codificar, consolidar, retener, almacenar, recuperar y evocar la información, que fue previamente registrada a través de los sentidos (Crespo, S. y Fernández, V. 2012). Además, es la habilidad para aprender y recordar informaciones basadas en experiencias importantes para la supervivencia, y se clasifica, en relación con el almacén de largo plazo, en memoria no declarativa (implícita) y memoria declarativa (explícita), la cual contiene información que el individuo puede expresar de forma verbal; además de permitir la evocación consciente de hechos, acontecimientos y estímulos específicos; son acontecimientos sobre los que se puede pensar y hablar y es accesible a múltiples sistemas de respuesta. Comprende dos subtipos de memoria: semántica y episódica; así, la memoria episódica se define como el almacenamiento de eventos donde se utiliza un código espacial y temporal que incluye el conocimiento de eventos y situaciones (Tulving, 1972; Ardila y Roselli, 2007); y la memoria semántica "es un tipo de memoria declarativa de la que depende nuestro conocimiento general sobre el mundo y del lenguaje” (Reber, Knowlton \& Squire, 1996; Bothelo, Acevedo, Conde y Tomaz, 2008).

\section{Alteraciones en el envejecimiento normal y la frontera con lo patológico}

Por su parte, Román Lapuente y Sánchez Navarro (1998); Roselli, Jurado y Matute (2008) y Ríos (2016), se refieren al envejecimiento como un proceso evolutivo, en la que en sus etapas finales se produce una disminución, en una u otra capacidad cognitiva, así como también asocian el declive cognitivo con el nivel educativo. En la investigación "Cambios neuropsicológicos asociados al envejecimiento normal" de Román y Sánchez (1998), el rendimiento en pruebas verbales, se rela- 
ciona a factores adversos, como por ejemplo, condiciones deficientes de salud, bajo nivel educativo o bajo coeficiente intelectual, que podrían estar produciendo un efecto negativo en el rendimiento en las pruebas de memoria, especialmente en personas muy mayores, lo cual podría estar relacionado con las capacidades o habilidades aprendidas durante la vida. Por otro lado, y en esta misma investigación, se habla de factores ambientales internos, por ejemplo el nivel de estrógenos, los cuales podrían estar relacionados con el funcionamiento cognitivo durante el envejecimiento normal, com también es abordado por Sarabia-Cobo (2009) en sus planteamientos. Así, Román Lapuente y Sanchez Navarro (1998), observan una tendencia de las mujeres mayores a presentar un mejor funcionamiento cognitivo, en función del retraso en la aparición de la menopausia, mostrando una cierta superioridad en las que aparece más tarde; es importante aclarar que no todas las funciones cognitivas disminuyen por igual.

Otros estudios han puesto de manifiesto que durante el envejecimiento aparece un déficit, tanto en la capacidad para resolver problemas, como en la toma de decisiones (Cronin \& Hof, 2004).

Así las cosas, las alteraciones que puedan presentarse en la atención del adulto mayor, están íntimamente relacionadas con la motivación que despierte la tarea que se esté realizando, y con las alteraciones perceptivas que pudieran estar relacionadas con la edad, de tal manera que en condiciones ambientales desfavorables, la atención sostenida podría debilitarse, mientras que en ambientes estimulantes y tareas de interés, podría lograrse una optimización de la atención. En los procesos demenciales, la atención no le permite al adulto mayor guardar la información, por tanto, la atención comienza a deteriorarse, junto con la memoria a corto plazo, y en la medida en que la enfermedad avanza, se vuelve más desatento y con mayor dificultad para sostener la atención en las actividades que realiza (Román y Sanchez, 1998). Así mismo, la capacidad para seleccionar los estímulos adecuados, para la realización de cada tarea, se va alterando progresivamente, hasta el ensimismamiento en etapas de deterioro avanzado. La secuencia del de- 
terioro de la percepción en la demencia, se inicia en el momento en que el paciente comienza a perder su capacidad para recordar; esto trae como consecuencia que el paciente comience a tener dificultades para interpretar correctamente y reconocer adecuadamente las situaciones, las personas y los objetos que se encuentran en su entorno (Sánchez y Pérez, 2008; Morgado, 2005).

\section{Relaciones neuroanatómicas con los cambios neurofuncionales}

El enlentecimiento en la velocidad de procesamiento se ve afectado por la disminución en la velocidad de la transmisión nerviosa, la cual se ha asociado con cambios neurodegenerativos en la sustancia blanca. Respecto a la memoria a corto plazo, aparece un deterioro de la memoria de trabajo, más específicamente del sistema ejecutivo central, lo que se ha asociado, tanto al enlentecimiento de la velocidad de procesamiento, como al deterioro de los lóbulos frontales. Por su parte, dentro de la memoria a largo plazo, aparece un déficit mayor de la memoria declarativa durante el envejecimiento. A su vez, dentro de este subsistema, declina más la memoria episódica, manteniéndose relativamente la semántica.

Estos déficits se han asociado con los cambios que aparecen en la corteza temporal, más específicamente con la pérdida neuronal de la formación hipocámpica, aunque también se han relacionado con los cambios a nivel talámico y áreas corticales, así como con una disminución colinérgica, catecolaminérgica y de fosfolípidos. Aunque todos estos cambios se producen durante el envejecimiento, no tienen por qué aparecer en un mismo sujeto, dando lugar a una serie de diferencias individuales dentro de la población de adultos mayores (Román y Sanchez, 1998; Tomaz y Costa, 2001; García, Fusari y Ellgring, 2008). En este sentido, como lo abordan Ríos y Jiménez (2015), las bases estructurales de la vida y el funcionamiento del ser humano son las Redes Neuronales que se configuran a partir de la experiencia y las interacciones con diversos factores intrínsecos o extrínsecos al organismo, esto conllevaría, según lo abordan los autores, a una adaptación cerebral 
individual que se soporta en una estructura común a todo individuo, el sistema nervioso central; la evidencia de esto resultaría, para el caso de la investigación, en la asociación que existe entre la información y la carga emocional, al momento de codificar un contenido o suceso.

\section{Influencia de las emociones sobre la codificación de la información}

En este sentido, y en relación con elementos que favorecerían la codificación y recuerdo de la información, se ha abordado a las emociones, que, para Ríos (2016), "están significativamente relacionadas con la alteración o conservación, en la codificación y evocación de la información". Se pueden considerar entonces, a las respuestas emocionales, como una característica de los procesos mnemónicos (Kesner, 1992), por lo cual uno de los factores influyentes, en el almacenamiento de la información, es el supuesto en el que la emoción está ligada al tipo de recuerdo. Esto acentúa las diferencias entre individuos, en la forma en que se relata y expresa un suceso o se recobra la información codificada, donde el contexto de adquisición de los mismos es crucial, es así, que como lo refieren Ríos y Jiménez (2015):

...darle carácter biológico al pensamiento y las emociones, no es sinónimo de tomar al ser humano como una máquina básica de estímulos y respuestas; por el contrario, dentro de la objetividad científica del sistema nervioso central, la subjetividad parte de las diferencias entre sujetos en las redes sinápticas, de los aprendizajes y experiencias propias que se acentúan y configuran sobre una base neuronal y que en mayor medida dependen del ambiente...

Las emociones se consideran, entonces, como estados psicológicos complejos que alcanzan aspectos cognitivos, fisiológicos y conductuales. Desde una mirada biológico-evolucionista, las emociones son estados del organismo que activan diferentes respuestas primitivas, ante situaciones como supervivencia, reproducción, defensa y apareamiento (Aguado, 2002). Se tiene entonces, que las emociones son respuestas que se manifiestan físicamente en la persona, alertando algún suceso en particular que nos puede afectar de manera positiva o negativa, 
como una reacción estimulante a un momento de dificultad, o de alerta ante el peligro. Ahora bien, se puede entender un estado emocional como la percepción que tiene el cerebro a diferentes estados estimulantes, con el fin de programar inconscientemente nuestra memoria a los estados de percepción.

\section{Triada funcional del adulto: emociones, memoria y envejecimiento}

Investigaciones, como la realizada por García, Fusari, y Ellgring (2008), han reportado un deterioro en el procesamiento emocional, tanto en el envejecimiento normal como en el patológico, en especial en las emociones negativas (ira, miedo y tristeza). Esto se evidencia, fundamentalmente, por los daños en la amígdala, y en zonas específicas de la corteza prefrontal.

Siguiendo esta línea, se puede decir que la memoria emocional ha de pensarse como la consecuencia de la adquisición, almacenamiento y evocación del accionar emocional (Santiago de Torres, 1999), producido durante una experiencia concreta (Cahill \& McGaugh, 1995; Cahill, 1999; De Torres, Tornay y Gómez, 2006). Por lo anterior, se problematiza, esencialmente, la separación de la relación simultanea de la experiencia cognitiva y la vivencia emocional (Conde, Prada, Martinez, Bothelo y Becerra, 2007).

Pocos estudios han abordado la relación existente entre memoria y los contenidos emocionales, y cómo estos influyen en la recuperación de la información; en este punto, se resalta el estudio de Bothelo, Martinez, Conde, Prada y Bezerra (2004), en el cual se evaluó una muestra colombiana, con la prueba auditivo-visual, para memoria declarativa, asociada a contenido emocional, evidenciando diferencias significativas en el recuerdo libre. Los participantes que presenciaron la versión emocional, recordaron más detalles que los de la versión neutra, e igualmente asignaron un valor superior a la historia emocional, lo que confirma una estrecha relación entre el componente emocional y la consolidación de la memoria. La activación, los eventos y los alertamientos emocionales, de acuerdo a la situación y las características, pueden ser recordados con claridad, detalle y potencian un incremento 
de la memoria declarativa, es decir, la activación emocional; de acuerdo a la experiencia, el almacenamiento se considera el resultado y la evocación de la memoria emocional (Christianson, 1992; Reisberg \& Hertel, 2004).

Por otra parte, numerosos estudios (Gaugh \& Gold, 1989; Heuer \& Reisberg, 1990; Burke, Heuer, \& Reisberg, 1992; Lang, Simons \& Balban, 1997; Kazui et al., 2000; Bothelo et al., 2004, De Torres et al., 2006; Broche \& Herrera, 2011), que abordan la evaluación de las respuestas fisiológicas, asociadas a la memoria emocional, han evidenciado la influencia de la activación emocional, sobre la retención de la información, causado por la experiencia vivida; estos estudios, que utilizaron la prueba auditivo visual, con imágenes impactantes para el paciente, demostraron un incremento de la memoria declarativa inducida, especialmente por los contenidos alertadores presentes en tales historias.

Cuando las características de los estímulos han sido catalogadas como "alertadoras" del evento presentado, con frecuencia se asocian a un mejor recuerdo, no sólo con una mayor claridad, sino también con mayor detalle. Así también, estudios con población de tercera edad (Blasco y Melendez, 2006), demuestran que tienen mayores recuerdos de su infancia, específicamente de eventos que marcaron su vida, asociado a la memoria declarativa. Frecuentemente, las personas que se han expuesto a sucesos traumáticos o a experiencias emocionales extremas, es decir, a contenidos emocionales fuertes, se logra evocar con mayor facilidad el recuerdo, en referencia a la memoria declarativa (Agudelo, 2002; Bothelo et al., 2004; Blasco y Melendez, 2006; Conde et al., 2007). Este efecto, se ha obtenido también, al comparar, tanto el recuerdo de palabras neutras y emocionales, como su reconocimiento (Fernández, Granero, Barrantes y Capdevila, 1997; LaBar, Gatenby, Gore, LeDoux \& Phelps, 1998).

Este conjunto de evidencias sugiere que la superioridad de la información emocional, respecto a la neutra en la retención, es un fenómeno importante, que se obtiene claramente cuando el sujeto se centra en el significado de los estímulos durante su codificación, y que se observa, tanto con material verbal, como con imágenes; lo cual debe ser algo intrínseco a la información emocional, y no relacionado con su discri- 
minabilidad. Los estímulos emocionales, son mejor recordados que los neutros, incluso aunque el sujeto no haya atendido explícitamente a su valencia afectiva durante la codificación (Ferré, 2002).

Es crucial tener en cuenta que lo emocional y lo cognitivo no pueden separarse, ya que ambos tienen una estrecha relación bidireccional (Broche y Herrera, 2011). Por ende, se hace relevante abordar, en esta investigación, la relación entre estos conceptos, para esclarecer su afectación desde diferentes patologías, y en particular la Enfermedad de Parkinson, buscando ir más allá de lo motor y lo cognitivo, ya que diversos estudios han mostrado indicios de ello y su relación con el envejecimiento tanto normal como patológico, lejos de abordar específicamente la demencia de Párkinson.

\section{Metodología}

Esta investigación es de enfoque cuantitativo, con diseño descriptivo-correlacional, y un modelo para selección de muestra no probabilístico y voluntario (Hernádez, Fernández y Baptista, 2014). Los datos obtenidos en la recolección de información, producto del protocolo aplicado, se analizaron mediante estadística descriptiva-correlacional, empleando el software estadístico SPSS versión 22; se ejecutó prueba de identificación de normalidad de los datos de Kolmogorov-Smirnov, con corrección de significación de Lilliefors; pruebas paramétricas a t Student, no paramétricas a U de Mann-Whitney; coeficientes de correlación de Pearson y Spearman; Chí cuadrado; frecuencias y descriptivos generales.

\section{Sujetos}

La muestra estuvo conformada por 40 adultos, divididos en 2 grupos, de 20 personas cada uno; un grupo clínico, diagnosticado por neurología con enfermedad de Parkinson, y un grupo control sin alteraciones neuropsicológicas, mentales o físicas, diferentes a las del envejecimiento normal, y en condición par al grupo clínico, en lo relacionado con edad, género y escolaridad, es decir, por ejemplo, si un adulto clínico tiene 70 
años, género femenino y universitario, su par no clínico cumple estas mismas características. Ambos grupos, con edades comprendidas entre los 60 y los 80 años de edad. A su vez, los grupos se subdividieron aleatoriamente en 2 grupos, de acuerdo al tipo de versión que presenciarían de la prueba auditivo-visual (emocional o neutra).

\section{Instrumentos}

La investigación cofiguró un protocólo de evaluación, la cual se realizó mediante la aplicación de los test, sub test y pruebas seleccionadas; GDS: escala de depresión geriátrica (Yesavage \& Sheikh, 1986); prueba de memoria declarativa, asociada a contenido visual Auditivo-Visual: Versión colombiana (Bothelo et al. 2004); Neuropsi: evaluación neuropsicológica breve en español (Otrosky, Ardila \& Roselli, 2000); Curva de memoria: California verbal Learning test (CVLT) (Woods, Delis, Scott, Kramer, \& Holdnack, 2006); Test stroop: test de colores y palabras Stroop elaborado por Charles Golden (Golden, 2010); Trail Making Test: test de trazos (Reitan, 1958); y el test de reconocimiento de emociones en rostros (Baron-Cohen, Wheelwright \& Jollife, 1997).

\section{Procedimiento}

Las pruebas, en su aplicación, se realizaron en tres sesiones individuales, de una hora aproximadamente; al inicio de cada una se verificó el estado atencional y emocional del participante, además de controlar las variables contextuales y ambientales, que pudieran interferir en la aplicación de las pruebas; seguido a esta inspección, se inició la aplicación de los test y sub test que conformaron el protocolo, distribuidas a lo largo de las 3 sesiones. Se aplicó la versión de la prueba auditivo-visual al participante, según el subgrupo al que había sido designado aleatoriamente; el resto del protocolo de evaluación, se aplicó por igual a todos los sujetos. 


\section{Consideraciones éticas}

Los procedimientos realizados en la presente investigación se diseñaron de conformidad con las directrices estipuladas por el Ministerio de Salud de Colombia (Resolución No 8430 de 1993), sobre la investigación con participantes humanos y el nivel de riesgo que implica; por tanto, el estudio se cataloga como riesgo mínimo, debido a que no puso en riesgo la integridad física o psicológica de los participantes. Adicionalmente, la investigación se ajustó a las disposiciones estipuladas por el Código Ético y Deontológico del Psicólogo (Ley 1090, 2006), en relación con la protección de la identidad, buen nombre, participación voluntaria y fines estrictamente investigativos de los resultados del estudio. Así, la presente investigación se realizó bajo la aprobación de los participantes y su acudiente, a través del consentimiento informado; previo a la realización del procedimiento de aplicación. Así, se diligenció el consentimiento informado, firmado por cada participante y/o representante legal. Para lo anterior, se les explicó en qué consistía el procedimiento y qué implicaciones tenía el hecho de aceptar participar en el mismo, el carácter voluntario de la participación y la posibilidad de abandono con previo aviso del mismo.

\section{Resultados}

Los datos descriptivos, realizados a ambos grupos, reflejan que para la variable edad, en ambas muestras, se tiene como media 69 años, con desviación estándar (DE) de 10,86. Al verificar el estado cognitivo de los participantes a conformar cada una de las muestras, se halla que el grupo control presenta una media de 93,13 puntos (DE 10,186), en la prueba Neuropsi, al evaluar el estado neuropsicológico general, ubicándose en el rango normal de la prueba. Por otro lado, el grupo con enfermedad de Parkinson obtiene una puntuación media de 78,08 (DE 25,027), ubicándose en un rango de deterioro cognitivo moderado, según la escala. Tras la evaluación del estado atencional de ambos grupos, se encontró que estos no presentan diferencias significativas estadísticamente en los test aplicados para realizar esta valoración. 
Tabla 1. Datos prueba auditivo visual, memoria declarativa asociada a contenido emocional

\begin{tabular}{|c|c|c|c|c|c|c|c|c|c|c|c|c|}
\hline \multirow[b]{2}{*}{ Criterio } & \multicolumn{5}{|c|}{ Versión Neutra } & \multicolumn{7}{|c|}{ Versión Emocional } \\
\hline & & M & $\mathrm{DE}$ & Q1 & Q3 & M & $\mathrm{DE}$ & Q1 & Q3 & Sig.1 & Sig.2 & Sig.3 \\
\hline \multirow{2}{*}{ Fase 1} & G1 & 2.90 & 1.19 & 2.75 & 4.00 & 5.00 & 2.70 & 3.75 & 7.00 & \multirow{2}{*}{$0.007^{* * t}$} & \multirow{2}{*}{$0.702^{u}$} & $0.018^{* t}$ \\
\hline & G2 & 3.60 & 2.27 & 1.75 & 6.00 & 2.00 & 1.56 & 0.75 & 3.00 & & & $0.083^{t}$ \\
\hline \multirow{2}{*}{ Fase 2} & G1 & 2.90 & 1.10 & 2.00 & 4.00 & 5.20 & 3.04 & 2.75 & 7.75 & \multirow{2}{*}{$0.050^{* t}$} & \multirow{2}{*}{$0.181^{t}$} & $0.040^{* \mathrm{t}}$ \\
\hline & G2 & 2.10 & 1.44 & 1.00 & 3.00 & 2.80 & 1.93 & 1.00 & 4.25 & & & $0.372^{t}$ \\
\hline \multirow{2}{*}{ Fase 3} & G1 & 3.20 & 1.54 & 2.00 & 4.25 & 4.30 & 1.88 & 3.00 & 5.25 & \multirow{2}{*}{$0.000^{* * * u}$} & \multirow{2}{*}{$0.511^{\mathrm{u}}$} & $0.172^{t}$ \\
\hline & G2 & 2.50 & 1.58 & 0.75 & 4.00 & 0.80 & 1.13 & 0.00 & 2.00 & & & $0.016^{* u}$ \\
\hline \multirow{2}{*}{$\begin{array}{l}\text { Total } \\
\text { Fases }\end{array}$} & G1 & 9.00 & 2.78 & 6.75 & 11.25 & 13.20 & 5.67 & 8.50 & 17.25 & \multirow{2}{*}{$0.002^{* * t}$} & \multirow{2}{*}{$0.654^{t}$} & $0.050^{* t}$ \\
\hline & G2 & 8.20 & 4.80 & 3.75 & 12.50 & 5.70 & 3.02 & 3.00 & 8.50 & & & $0.180^{t}$ \\
\hline \multirow{2}{*}{ Cuestionario } & G1 & 9.10 & 2.68 & 7.00 & 11.25 & 13.90 & 6.04 & 8.50 & 17.75 & \multirow{2}{*}{$0.004^{* * t}$} & \multirow{2}{*}{$0.611^{t}$} & $0.034^{* t}$ \\
\hline & G2 & 8.20 & 4.80 & 3.75 & 12.50 & 7.00 & 2.70 & 5.00 & 9.50 & & & $0.502^{t}$ \\
\hline \multirow{2}{*}{$\begin{array}{l}\text { Valor } \\
\text { Emocional }\end{array}$} & G1 & 2.70 & 2.21 & 0.75 & 4.25 & 8.30 & 2.54 & 5.00 & 10.00 & \multirow{2}{*}{$0.160^{u}$} & \multirow{2}{*}{$0.328^{t}$} & $0.001^{* * * u}$ \\
\hline & G2 & 3.80 & 2.65 & 1.50 & 5.50 & 7.40 & 2.95 & 6.50 & 9.00 & & & $0.502^{u}$ \\
\hline \multirow{2}{*}{ Felicidad } & G1 & 1.60 & 2.54 & 0.00 & 3.50 & 0.60 & 1.57 & 0.00 & 0.25 & \multirow{2}{*}{$0.487^{\mathrm{u}}$} & \multirow{2}{*}{$0.534^{u}$} & $0.305^{u}$ \\
\hline & G2 & 0.80 & 1.39 & 0.00 & 2.00 & 1.90 & 3.60 & 0.00 & 3.25 & & & $0.815^{u}$ \\
\hline \multirow{2}{*}{ Sorpresa } & G1 & 1.70 & 2.71 & 0.00 & 2.75 & $5 \cdot 70$ & 3.62 & 2.75 & 10.00 & \multirow{2}{*}{$0.715^{t}$} & \multirow{2}{*}{$0.239^{u}$} & $0.010^{* * u}$ \\
\hline & G2 & 2.90 & 2.37 & 0.00 & 5.00 & 5.10 & 3.60 & 1.50 & 8.25 & & & $0.125^{t}$ \\
\hline \multirow{2}{*}{ Miedo } & G1 & 1.20 & 2.53 & 0.00 & 2.00 & 6.10 & 3.44 & 3.50 & 9.25 & \multirow{2}{*}{$0.045^{* t}$} & \multirow{2}{*}{$0.370^{u}$} & $0.003^{* * u}$ \\
\hline & G2 & 1.90 & 2.37 & 0.00 & 4.25 & 2.80 & $3 \cdot 39$ & 0.00 & 4.75 & & & $0.634^{u}$ \\
\hline \multirow{2}{*}{ Rabia } & G1 & 1.00 & 2.53 & 0.00 & 0.50 & 4.10 & 4.14 & 0.00 & 9.25 & $0.480^{u}$ & & $0.025^{* u}$ \\
\hline & G2 & 0.20 & 0.63 & 0.00 & 0.00 & 3.30 & 4.47 & 0.00 & 9.25 & 0.400 & 0.503 & $0.044^{* u}$ \\
\hline & G1 & 2.20 & 3.01 & 0.00 & 2.50 & 9.60 & 0.84 & 9.50 & 10.00 & & & $0.000^{* * * u}$ \\
\hline Tristeza & G2 & 2.80 & 3.29 & 0.00 & 4.50 & 8.60 & 3.09 & 8.75 & 10.00 & $0.400^{\mathrm{u}}$ & $0.676^{u}$ & $0.005^{* * u}$ \\
\hline
\end{tabular}

Nota: G1: envejecimiento normal, G2: Enfermedad de Parkinson M: media, DE: desviación estándar, Q: cuartil, t: T-Student, u: U de Mann Whitney, ${ }^{*} \mathrm{p} \leq 0.05,{ }^{* *} \mathrm{p} \leq 0.01,{ }^{* * *} \mathrm{p} \leq 0.001$, Sig $^{1}$ : diferencias entre grupos en relación a la versión emocional, $\mathrm{Sig}^{2}$ : diferencias entre grupos en relación a la versión neutra, Sig3: diferencias entre versiones en relación al grupo.

Fuente: Autores 
Los datos contenidos en la Tabla 1, permiten identificar, en su análisis, diferencias significativas desde el punto de vista estadístico, entre las versiones de la prueba presenciadas (Sig.3) y el grupo, principalmente para los criterios fase 1 , fase 2 , total fases, cuestionario y valor emocional; en esta última, y en fase 1, el grupo 2, es decir, el de pacientes con enfermedad de Parkinson, reportó mayor carga emocional y mayores puntuaciones en la prueba, en relación con las versiones presenciadas (emocional o neutra). Se encuentran diferencias intragrupo, en relación con las emociones sorpresa, miedo, rabia y tristeza para el grupo 1; estas dos últimas, compartidas con el grupo 2.

De igual forma (tabla 1), no se encuentran diferencias significativas entre los dos grupos para quienes presenciaron la versión neutra de la prueba de memoria declarativa asociada a contenido emocional (Sig. 2). En lo que respecta a la versión emocional (Sig. 1), se hallaron diferencias en cuanto a la información evocada para los criterios fase 1 , fase 2 , fase 3 , total fases, cuestionario y miedo, siendo esta última la única emoción diferente entre los dos grupos, con puntuaciones inferiores para el grupo 2 en todos los criterios en los diferentes niveles de p. Para ambos grupos, las mayores puntuaciones fueron obtenidas por quienes presenciaron la versión emocional de la prueba, en todos los criterios contenidos en la tabla 1 , aun cuando no todos con diferencias significativas.

Tabla 2. Asociaciones entre criterios test emocionales y de memoria

\begin{tabular}{|c|c|c|c|c|c|c|c|c|c|c|}
\hline & \multicolumn{5}{|c|}{ Versión Neutra } & \multicolumn{5}{|c|}{ Versión Emocional } \\
\hline Criterio & M & DE & Q1 & Q3 & $\mathbf{r 1}$ & M & DE & Q1 & Q3 & r2 \\
\hline $\begin{array}{l}\text { Tot a l } \\
\text { Fases }\end{array}$ & 8.60 & 3.844 & 5.25 & 11.75 & \multirow{2}{*}{$0,695^{* \mathrm{~s}}$} & 9.45 & 5.862 & 5.00 & 14.00 & \multirow{2}{*}{$0,615^{* e}$} \\
\hline МСР & 5.50 & 3.236 & 3.25 & 8.00 & & 4.85 & 2.852 & 3.00 & 6.75 & \\
\hline
\end{tabular}




\begin{tabular}{|c|c|c|c|c|c|c|c|c|c|c|}
\hline $\begin{array}{c}\text { Cues- } \\
\text { tionario } \\
\text { A-V }\end{array}$ & 8.65 & 3.815 & 5.50 & 11.75 & \multirow[b]{2}{*}{$0,565^{* e}$} & 10.45 & $5 \cdot 772$ & 5.25 & 14.00 & \multirow[b]{2}{*}{$0,541^{* e}$} \\
\hline $\begin{array}{c}\text { Clave } \\
\text { Semán- } \\
\text { tica } \\
\text { (MLP) }\end{array}$ & 6.80 & 3.139 & 6.00 & 8.75 & & 5.10 & $3 \cdot 323$ & 3.25 & 7.75 & \\
\hline $\begin{array}{c}\text { Valor } \\
\text { Emocio- } \\
\text { nal A-V }\end{array}$ & 3.25 & 2.447 & 1.25 & 5.00 & \multirow{2}{*}{$0,185^{\mathrm{e}}$} & 7.85 & 2.720 & 5.50 & 10.00 & \multirow{2}{*}{$0,286^{\mathrm{e}}$} \\
\hline $\begin{array}{c}\text { Emocio- } \\
\text { nes en } \\
\text { Rostros }\end{array}$ & 14.30 & 2.386 & 13.00 & 16.00 & & 14.55 & 2.819 & 14.25 & 16.00 & \\
\hline
\end{tabular}

Nota: M: media, DE: desviación estándar, Q: cuartil, r: correlación, ${ }^{*} \mathrm{p} \leq 0.05,{ }^{* *} \mathrm{p} \leq 0.01$, e: Pearson, s: Spearman, MCP: memoria a corto plazo, A-V: memoria auditivo visual, MLP: memoria a largo plazo.

Fuente: Autores

En referencia con las correlaciones presentadas en la tabla 2, se encuentra que los criterios: total fases y MCP, se correlacionan de forma directa y proporcional, tanto para quienes presenciaron la versión neutra, como la emocional, en la prueba de memoria declarativa, asociada a contenido emocional, independientemente de la condición o no de enfermedad de Parkinson; de igual forma, ocurre en la relación entre el cuestionario A-V y clave semántica MLP, ambas correlaciones a nivel de $\mathrm{p} \leq 0.05$; es decir, a mayor desempeño en una variable, la otra aumentaba en la misma proporción. De otro lado, aun cuando los criterios valor emocional A-V y emociones en rostros, no presentan correlación significativa, su asociación es positiva.

\section{Discusión}

Los análisis realizados, producto de los datos obtenidos en la investigación, ponen de manifiesto diferencias significativas entre el funcionamiento de la memoria y la influencia de las emociones sobre ésta; dentro del envejecimiento normal, y en comparación con el desarrollo 
de la enfermedad de Parkinson, estos datos concuerdan y divergen en alguna medida con los datos reportados en la literatura científica.

Los pacientes con enfermedad de Parkinson presentan menores alteraciones en la memoria inmediata, aún cuando su memoria, a corto plazo y a largo plazo, se ve comprometida en relación con la evocación de información visual y verbal; estos datos concuerdan con lo expuesto por Muñiz y Rodríguez (2007), quienes plantean que la memoria inmediata se encuentra preservada en estos pacientes, y, a su vez, presentan un déficit en el recuerdo de corto y largo plazo, para ambos canales de información. Sin embargo, la investigación aquí desarrollada encuentra que, cuando la codificación de la información se realiza bajo contenidos emocionales significativos, la memoria se ve potenciada en la cantidad y calidad de la información recordada, en los almacenes de corto y largo plazo, lo cual se asemeja a los hallazgos de estudios realizados por Bothelo et al. (2004), quienes han reportado la influencia de las emociones, sobre la memoria, principalmente de largo plazo para almacenes declarativos.

Lo anterior, supone relación con lo expuesto por Bradley, Lang y Cuthbert (1993); Burke et al. (1992); Cahill (1999); Cahill y McGaugh (1995); Heuer y Reisberg (1990); Lang (1995); Phelps, LaBar y Spencer (1997); y McGaugh (2000), que aunque aquí no se reporta directamente reacciones fisiológicas, es posible suponer, como lo abordan estos investigadores, que dichas respuestas fisiológicas, vinculadas a la memoria emocional, ponen de manifiesto la influencia de los contenidos emocionales, sobre la codificación y almacenamiento de la información en la memoria de corto plazo.

Tanto los pacientes con enfermedad de Parkinson, como los de envejecimiento normal, que conformaron la muestra de la investigación, recordaron mayor cantidad de información, evidenciada a partir de la evocación del material contenido en la memoria declarativa, entre quienes presenciaron la versión emocional de la prueba auditivo visual, en comparación con aquellos que evidenciaron la versión neutra; lo anterior, concuerda con estudios realizados, en diversas poblacio- 
nes, sobre la influencia de las emociones en la memoria, por Bothelo, Albarracin, Chona, Conde y Tomaz (2008), Bothelo et al. (2004); y Conde et al. (2007); autores que plantean un significativo efecto en la memoria, inducido por la carga emocional en el material evocado.

Por otra parte, Bothelo et al. (2008), plantean que emociones como tristeza y miedo, priman como influyentes al momento de evocar la información, entre quienes presenciaron la versión emocional. En relación con esto, la investigación aquí presentada, encuentra que contenidos relacionados con el miedo, se asocian, en mayor medida, a la codificación y almacenamiento de la información en el envejecimiento normal, no así en la enfermedad de Parkinson; adicional a ello, tristeza y "rabia" se asocian, por igual, con el envejecimiento normal, como patológico; de otro lado, estos mismos autores refieren que la emoción felicidad fue asociada significativamente entre quienes presenciaron la versión neutra; en relación con esto, no es posible coincidir con este planteamiento, puesto que ni el grupo control ni el clínico, del estudio aquí condensado, evidencia asociación significativa de esta emoción con la cantidad de información evocada para ninguna de las dos versiones de la prueba.

En síntesis, estos hallazgos refieren la influencia del vínculo emocional de una situación contextual que promueve la codificación, el almacenamiento y la evocación de la información, potenciándose la memoria declarativa; lo anterior, supone concordancia con postulados formulados por Bothelo et al. (2004), quienes refieren que los contenidos emocionales de la memoria se suscitan a partir de características específicas de la situación emocional, que son recordadas más claramente y en mayor detalle.

En este sentido, es clara la influencia de las emociones sobre la memoria; sin embargo, estas actúan de forma diversa en la codificación de la información, lo cual dependerá del recuerdo de la misma, a partir de los vínculos suscitados y establecidos por el sujeto, en el momento emocional o carente de ello, lo cual varía entre cuadros de envejecimiento normal o patológico, principalmente en referencia aquí a la enfermedad de Parkinson. 


\section{Conclusiones}

Los análisis obtenidos en investigación, ponen de manifiesto la influencia de las emociones sobre la memoria declarativa en adultos con enfermedad de Parkinson, y las principales emociones asociadas a esto. La prueba de memoria declarativa, asociada a contenido emocional, es un instrumento sensible en la detección del rol de las emociones sobre la memoria declarativa, en sus diferentes tiempos de almacenamiento, así como las emociones que presentan mayor o menor influencia sobre estos.

Los adultos con enfermedad de Parkinson, que presenciaron la información con contenido emocional, obtuvieron una mayor tasa de recuerdo, que aquellos que obtuvieron información con emoción neutra; aun cuando los adultos con enfermedad de Parkinson recuperan mayor información, cuando se asocia a emociones, la cantidad de información recuperada es inferior a la de los adultos con envejecimiento normal; y la información carente de contenido emocional, se codifica y almacena, de igual manera, ante la condición de envejecimiento normal o patológico.

Las atribuciones generales de valor emocional de la información, son indistintas a contenidos neutros y emocionales en el cuadro patológico; sin embargo, dentro del envejecimiento normal se atribuye mayor carga emocional a la información almacenada en la memoria declarativa; en los adultos con enfermedad de Parkinson, emociones como felicidad, sorpresa y miedo, no se asocian con la información evocada.

Cuando la información, tanto visual como verbal, se codifica bajo la carencia de emociones asociadas, la recuperación de ésta, es inferior en cuanto a tasa de recuerdo, e indistinta al envejecimiento normal o patología de Parkinson. De otro lado, cuando la información contextual se codifica en la memoria declarativa, ante emociones como el miedo, éste influye de manera positiva en el recuerdo de los contenidos almacenados.

Aun cuando las emociones positivas se asocian a los contenidos de la memoria, estas tienen menor influencia en la recuperación de la in- 
formación, actuando de manera indiferente ante una condición o no patológica del envejecimiento. Dentro de la condición de Parkinson, emociones como tristeza y rabia se relacionan en mayor medida a los contenidos de la memoria declarativa, tal como ocurre en el envejecimiento normal.

En relación con los canales por los cuales se obtiene la información, y considerando que las imágenes presenciadas, tanto para la versión neutra, como para la emocional, son las mismas, modificando su relato; es de resaltar la influencia de la información verbal, sobre la información visual, interfiriendo en mayor medida sobre el almacenamiento de la información. De otro lado, cuando se accede a claves semánticas, los adultos con enfermedad de Parkinson recuperan más fácilmente la información, siempre y cuando éstas se hayan codificado bajo la presencia de emociones principalmente negativas.

Las atribuciones de valor emocional, a una situación contextual, se relacionan al recuerdo, y son independientes a la habilidad de reconocer expresiones faciales, contenidas en rostros dentro de la Enfermedad de Parkinson. Por otra parte, es crucial la codificación de la información en la memoria de corto plazo para el posterior registro en los almacenes declarativos de largo plazo.

La enfermedad de Parkinson, aun cuando compromete principalmente funciones motoras, y en menor medida cognitivas, en relación con otras demencias, presenta alteraciones en el funcionamiento de la memoria, y se ve favorecida cuando la codificación de la información se presenta en un contexto con carga emotiva, que aunque independiente al tipo de emoción, las emociones negativas tienen mayor influencia.

Cuando se aborda el deterioro cognitivo desde el envejecimiento, la Enfermedad de Parkinson presenta menores alteraciones y desempeños cercanos a los del envejecimiento normal, en lo que a memoria y atención refiere; sin embargo, puede considerarse en esta enfermedad, la presencia de deterioro cognitivo leve, más que un cuadro demencial, por lo menos en los primeros años de evolución de la patología, lo cual puede suscitar hablar de estadíos del deterioro cognitivo en el Parkinson. 
De otro lado, desde la literatura científica y la práctica clínica, aun cuando los registros por neuroimagen no evidencian alteraciones estructurales en el cerebro del adulto mayor, diferentes a las usuales dentro del envejecimiento normal, áreas involucradas en el desarrollo de los procesos neuropsicológicos pueden verse comprometidas en funcionalidad; y aquellas que se ven afectadas por cuadros degenerativos, y relacionadas con funciones como la memoria, pudieran presentar menor nivel de alteración cuando, contenidos emocionales, se encuentran asociados en el momento de la codificación y posterior almacenamiento de la información. El abordaje de estos contenidos temáticos y estudios del funcionamiento cerebral deben orientarse desde la neuropsicología, entendida ésta como una disciplina científica de las neurociencias que se involucra en el estudio del sistema nervioso central (SNC) y los productos de actividad cerebral, como el pensamiento, la cognición, las emociones y la conducta, ante la presencia o no de cuadros patólogicos o lesiones estructurales, valiendose de la comprensión fisiológica y anatómica del SNC y sus interacciones con la biología general del sujeto.

\section{Agradecimientos}

A los participantes de la investigación, a las instituciones y familias que aceptaron participar en el estudio, propiciando espacios y dedicando su tiempo para la aplicación del protocolo de pruebas. A aquellas personas que entienden la importancia de compartir el conocimiento, y facilitaron la búsqueda de la información científica; igualmente al asesor temático y metodológico por su apoyo y asesoramiento en el desarrollo de la presente investigación.

\section{Limitaciones}

El poco material científico reciente limita visializar un panorama discutible sobre las variables y temáticas aquí analizadas y la población abordada con contexto actual; por lo que se hace limitado encontrar estudios recientes que contemplen una metodología similar a la aquí em- 
pleada o a los constructos considerados; así mismo, la mayoría de las investigaciones publicadas se limitan en arrojar resultados y conclusiones generales en cuanto a la conducta, cognición o las emociones como resultado de la influencia del envejecimiento normal o cuadros patológicos tipo Parkinson, por lo que resultados específicos hallados en esta investigación son contemplados como conclusión al no tener referente para discutir. De otro lado, aún cuando las muestras empleadas eran homólogas en criterios demográficos y cronológicos, se encuentran reducidas en cantidad de sujetos, por lo que a futuras investigaciones el empleo de una muestra más amplia aumentaría la confiabilidad en la generalización de los datos, más allá de la población local de referencia.

\section{Referencias}

Aguado, L. (2002). Procesos cognitivos y sistemas cerebrales de la emoción. Revista Neurologica, 34 (12), 1161-1170. Recuperado de www.neurologia.com/sec/ resumen.php?id=2002079.

Ardila, A. y Rosselli, M. (2007). Neuropsicología Clínica. Ed. Manual Moderno. México.

Baron-Cohen, S., Wheelwright, S. \& Jollife, T. (1997). Is there a "lenguaje of the eyes"? Evidence from normal adults, and adults with autismo or Asperger Syndrome. Visual Cognition, 4 (3), 311-331. doi: 10.1080/713756761.

Blasco, S. y Melendez, J. C. (2006). Cambios en la memoria asociados al envejecimiento.Geriatrika, 22(5), 179-185. Recuperado de www.uv.es/ melendez/ envejecimiento/memoriayvejez.pdf.

Bothelo de Oliveira, S., Albarracin, A. P., Chona, B. H., Conde, C. A. y Tomaz, C. (2008). Estudio correlacional entre memoria declarativa y una prueba psicométrica en una muestra de adolescentes escolares de la ciudad de Bucaramanga. Revista Colombiana Medica, 39(3), 60-70. Recuperado de www.scielo.org. co/pdf/cm/v39s3/v39s3a9.pdf. 
Bothelo De Oliveira, S., Martinez Garrido, L. M., Conde Cotes, C. A., Prada Sarmiento, E. L. y Bezerra Tomaz, C. A. (2004). Evaluacion de la memoria declarativa Asociada con contenido emocional en una muestra colombiana. Revista latinoamericana de Psicologia, 36 (2), 229-242. Recuperado de www.redalyc. org/articulo.oa?id=80536204.

Bothelo, S., Acevedo Prada, L. M., Conde Cotes, C. A., Fandiao Franky, J. Y Becerra Tomaz, C. A. (2008). Evaluacion de la memoria declarativa asociada al contenido emocional en pacientes lobectomizados. Revista Latinoamericana de Psicologia, 4O(2), 229-241. Recuperado de www.scielo.org.co/pdf/rlps/ v4on2/v40n2ao3.pdf.

Broche Pérez, Y. B. y Herrera Jimenez, L. F. (2011). Memoria de rostros y reconocimiento emocional: Particularidades en adultos mayores institucionalizados y que conviven con sus familiares. Neuropsicologia Latinoamericana, 3(3), 12-19. doi: 10.5579/rnl.2011.0078.

Bradley, M. M., Lang, P. J., \& Cuthbert, B. N. (1993). Emotion, novelty, and the startle reflex: habituation in humans. Behavioral neuroscience, 107(6), 970. doi: 10.1037/0735-7044.107.6.970.

Burke, A., Heuer, F. y Reisberg, D. (1992). Remembering emotional events. Memory \& Cognition, 2O(3), 277-290. doi: 10.3758/BFo3199665.

Cahill, L. y McGaugh, J. L. (1995). A Novel Demonstration of Enhanced Memory Associated with Emotional Arousal. En Consciousness and Cognition, 4(4), 410-421. doi: 10.3758/BFo3199665.

Cahill, L. (1999). A neurobiological perspective on emotionally influenced, long term memory. Seminars in Clinical Neuropsychiatry, 4(4), 266-273. doi: 10.153/ SCNPo0400266.

Casamitjana, C. F. (2007). Calidad de vida en pacientes con Enfermedad de Parkinson y estimulacion cerebral profunda. Medicina Interna de Mexico, 23(1), 7-14. Recuperado de new.medigraphic.com/cgi-bin/resumen.cgi?IDARTICULO=18283. 
Christianson, S. A. (1992). Emotional stress and eyewitness memory: a critical review. Psychol Bulletin, 112(2), 284-309. doi: 10.1037/0033-2909.112.2.284.

Conde Cotes, C. A., Prada Sarmiento, E. L., Martinez Garrido, L. M., Bothelo De Oliveira, S. y Becerra Tomaz, C. A. (2007). Evaluación de las manifestaciones autonómicas asociadas a la aplicación de una prueba auditivo-visual de memoria emocional en humanos. Universitas Psychologica, 7 (1), 109-124. Recuperado de www.scielo.org.co/pdf/rups/v7n1/v7n1ao9.pdf

Congreso de la República de Colombia. (2006). Ley 1090, por la cual se reglamenta el ejercicio de la profesión de Psicología, se dicta el Código Deontológico y Bioético y otras disposiciones. Recuperado de www.corteconstitucional.gov. co/relatoria/2007/C-832-07.htm.

Crespo-Santiago, D. y Fernández-Viadero, C. (2012). Cambios cerebrales en el envejecimiento normal y patologico. Revista Neuropsicologia, Neuropsiquiatría y Neurociencias, 12 (1), 21-36. Recuperado de: neurociencias.udea.edu.co/revista/?action=resumen\&id $=180$.

Cronin Golomb, A., \& Hof, P. R. (2004). Vision in Alzheimer's Disease. Suiza: Karger. De Torres, S., Tornay Mejías, F., y Gomez Milán, E. (2006). Procesos Psicologicos. México: McGraw-Hill.

Ferré Romeu, P. (2002). Recuerdo de imagenes emocionales y niveles de procesamiento. Psicothema, 14(3), 591-596. Recuperado de www.unioviedo.net/reunido/index.php/PST/article/view/7989.

Fernández Castro, J., Granero Pérez, R., Barrantes, N. y Capdevila, A. (1997). Estado de ánimo y sesgos en el recuerdo: papel del afecto. Psicothema, 9(2), 247258. Recuperado de www.unioviedo.net/reunido/index.php/PST/article/ view/7403.

García, S., Sauri Suárez, S., Meza Dávalo, E. y Lucino Castillo, J. (2008). Perspectiva historica y aspectos epidemiologicos de la Enfermedad de Parkinson. Medicina Interna de México, 24(1), 28-37. Recuperado de: new.medigraphic.com/ cgi-bin/resumen.cgi?IDARTICULO=19540. 
García Rodríguez, B., Fusari, A. y Ellgring, H. (2008). Procesamiento emocional de las expresiones faciales en el envejecimiento normal y patologico. Revista de Neurologia, 46(10), 609-617. Recuperado de www.neurologia.com/pdf/ Web/4610/z100609.pdf.

Gaugh, M., \& Gold. (1989). Emotion and memory. En R. J. Davidson, K. R. Scherer y H. H. Goldsmith. Handbook affective Sciences (255-287). New York, Estados Unidos: Oxford University Press.

Golden, C. J. (2010). Stroop test de colores y palabras. Madrid, España: Tea ediciones. Hernández Sampieri, C. R., Fernández Collado, C. y Baptista Lucio, P. (2014). Metodologia de la investigacion. Mexico: Mc Graw-hill.

Heuer \& Reisberg. (1990). The Handbook of Emotion and Memory. New York, Estados Unidos: Taylor and francis group.

Kazui, H., Mori, E., Hashimoto, M., Hirono, N., Imamura, T., Tanimukai, S., Hanihara, T. y Cahill, L. (2000). Impact of emotion on memory. The British Journal of Psychiatry, 177(4), 343-347. doi: 10.1192/bjp.177.4.343.

Kesner, R. P. (1992). Learning and memory in rats with an emphasis on the role of the amygdala. APA PsycNET. Recuperado de psycnet.apa.org/psycinfo/1992-97763-010.

LaBar, K. S., Gatenby, J. C., Gore, J. C., LeDoux, J. E. y Phelps, E. A. (1998). Human amygdala activation during conditioned fear acquisition and extinction: a mixed-trial fMRI study. Neuron, 2O(5), 937-945. doi: dx.doi.org/10.1016/ So896-6273(00)80475-4.

Lang, P. J. (1995). The emotion probe: studies of motivation and attention. APA PsycNET, 5O(5), 372. doi: 10.1037/0003-066X.50.5.372.

Lang, P. J., Simons, R. F. y Balaban (1997). Attention and Orienting: Sensory and Motivational Processses. New York: Lawrence Erlbaum Associates.

McGaugh. (2000) Memory a Century of Consolidation. Revista Neurociencias, 287, 248-251. doi: 10.1126/science.287.5451.248. 
Meza, M. (1998). Trastornos cognitivos en la enfermedad de Parkinson. Revista de neuro-psiquiatria, 61(1), 27-33. Recuperado de www.upch.edu.pe/vrinve/dugic/revistas/index. php/RNP/article/download/1446/1474.

Micheli, F. (2006). Enfermedad de parkinson y trastornos realcionados. Buenos Aires, Agentina: Médica Panamericana.

Ministerio de Salud de Colombia. (1993). Resolucion No 8430, por la cual se establecen las normas cientificas, técnicas y administrativas para la investigacion en salud. Recuperado de www.minsalud.gov.co/Normatividad_Nuevo/RESOLUCION\%208430\%20DE\%201993.pdf.

Morgado Bernal, I. (2005). Psicobiología del aprendizaje y la memoria: Fundametos y avances recientes. Revista Neurológica, 4O(5), 289-297. Recuperado de www. revneurol.com/sec/resumen.php?id=2005004.

Muñiz Casado, J. A. y Rodríguez Fernández, R. (2007). Deficit de memoria en pacientes con Enfermedad de Parkinson inicial. Mapfre medicina, 18(1), 39-45. Recuperado de www.mapfre.com/fundacion/html/revistas/medicina/v18sI/ pago2_o6_res.html.

Otrosky, F., Ardila, A. y Roselli, M. (2000). Evaluación neuropsicológica breve en español. Mexico: Publiingenio.

Parrao Díaz, T., Chaná Cuevas, P., Juri Chaverías, C., Kunstmann, C. y Tapia Núñez, J. (2005). Evaluacion del deterioro cognitivo en una poblacion de pacientes con enfermedad de parkinson mediante el test minimental Parkinson. Revista de neurologia, 4o(6), 339-344. Recuperado de www.neurologia.com/pdf/ Web/4006/so6o339.pdf.

Phelps, E. A. , LaBar, K. S. \& Spencer, D. D. (1997). Memory for emotional words following unilateral temporal lobectomy. Revista Brain And Cognition, 35(1), 85-109. doi:10.1006/brcg.1997.0929.

Reber, P., Knowlton, B. \& Squire, L. (1996). Studies of normal and abnormal memory in humans. En L. R. Squire y D. L. Schacter. Neurophicology and memory (pp. 803-813). New York, Estados Unidos: The Guilford Press. 
Reisberg, D. \& Hertel, P. (2004). Memory and emotion. New York, Estados Unidos: Oxford University Press.

Reitan, R. (1958). Validityn of the trail making test as an indicator of organic brain damage. Perceptual and motor skills, 8, 271-276. doi: 10.2466/ pms.1958.8.3.271.

Ríos Flórez, A. (2016). En el camino de la neuropsicología básica a la aplicación clínica en patología; memoria, emoción y envejecimiento. Revista Poiésis, 30, 72-78. Recuperado de http://www.funlam.edu.co/revistas/index.php/poiesis/ article/view/1847/1472.

Ríos Flórez, J. y Jiménez Zuluaga, P. (2015). Neurociencia y Psicoanálisis; Divergencias en la concepción biológica/subjetiva del ser humano. Revista Psicoespacios, 9(15), 263-282. Recuperado de http://revistas.iue.edu.co/index.php/ Psicoespacios.

Román Lapuente, F. y Sanchez Navarro, J. P. (1998). Cambios neuropsicológicos asociados al envejecimiento normal. Anales de Psicología, 14(1), 27-43. Recuperado de digitum.um.es/jspui/handle/10201/10150.

Roselli, M., Jurado, M. B. y Matute, E. (2008). Las Funciones Ejecutivas a través de la Vida. Revista Neuropsicología, Neuropsiquiatría y Neurociencias, 8(1), 2346. Recuperado de dialnet.unirioja.es/servlet/articulo? codigo $=3987451$.

Sánchez Gil, Y. I. y Pérez Martínez, V. T. (2008). El funcionamiento cognitivo en la vejez: atención y percepción en el adulto mayor. Revista cubana medicina general integral, 24(2), 1-8. Recuperado de scielo.sld.cu/scielo.php?pi$\mathrm{d}=$ So864-21252008000200011\&script=sci_arttext

Santiago de Torres, (1999). Emoción. En J. Santiago de Torres, F. Tornay Mejias y E. Gómez Milán. Procesos psicológicos básicos (215 - 229). Madrid, España: McGraw-Hill.

Sarabia-Cobo, C. M. (2009). Envejecimiento exitoso y calidad de vida. Su papel en las teorías del envejecimiento. Revista Gerokomos, 2O(4), 172-174. doi:10.4321/ S1134-928X2009000400005. 
Tomaz, C., \& Costa, J. C. (2001). Neurociência e memória. Humanidades, 48, 145-160. Recuperado de www.unifor.br/index.php?option=com_content\&view $=$ article\&id $=371 \&$ Itemid $=782$.

Tulving, E. (1972). Episodic and semantic memory. Episodic and semantic memory. New York, Estados Unidos: United Kingdom edition Academic Press. doi: 10.1017/So140525Xoo047257.

Vargas, A. (1997). Demencia y parkinson. Cuaderno de neurologia, 22, 02-06. Recuperado de escuela.med.puc.cl/paginas/publicaciones/neurologia/cuadernos/1997/pub_02_97.html.

Vila, E. y Barbero, I. (1992). Estudio neuropsicológico en la enfermedad de párkinson. Revista de psicología general y aplicada, 45(4), 439-442. Recuperado de dialnet.unirioja.es/servlet/articulo?codigo $=2378426$.

Woods, S., Delis , D., Scott, J., Kramer, J., \& Holdnack, J. (2006). The California verbal learning test- second edition: test-retest reliable change indices for the standard and alternate forms. Archives of clinical neuropsychology, 21(5), 413-420. doi: 10.1016/j.acn.2006.06.002.

Yesavage, J. \& Sheikh, J. (1986). Geriatric Depresion Scale, GDS Clinical Gerontology: Aguide to assessment and intrvention. New York: Th. doi: 10.1300/ Jo18v05no1_09. 